\title{
SATISFAÇÃO DOS PROFISSIONAIS DA ESTRATÉGIA SAÚDE DA FAMÍLIA NO MUNICÍPIO DE FORTALEZA (CE)
}

\section{Francisco Jander de Sousa Nogueira} sidade Federal do Piauí - Campus Parnaíba. Membro do Centro Brasileiro de Estudos de Saúde (CEBES), Brasil.

\section{Cesário Rui Callou Filho}

Mestre em Saúde Coletiva. Coordenador do Grupo de Estudo em Saúde (GESC) e Docente da Faculdade Vale do Salgado (FVS), Brasil.

\section{Caroline Antero Machado Mesquita}

Mestre em Saúde Coletiva. Membro do Grupo de Estudos em Saúde Coletiva (GESC), Brasil.

\section{Ilana Farias Andrade de Moura}

Enfermeira. Membro do Grupo de Estudo em Saúde Coletiva (GESC), Brasil.

\section{Edislane Silva Souza}

Enfermeira. Membro do Grupo de Estudo em Saúde Coletiva (GESC), Brasil.

\section{Fabrícia Salvador Bezerra}

Doutora em Saúde Pública. Membro do Grupo de Estudo em Saúde Coletiva (GESC), Brasil.
Docente Adjunto do Curso de Medicina da Univer-

RESUMO: Objetivou-se investigar os fatores que interferem na satisfação e insatisfação dos profissionais da Estratégia Saúde da Família (ESF). Trata-se de uma pesquisa avaliativa, do tipo transversal com abordagem quantitativa, realizada em 12 Unidades de Atenção Primária à Saúde (UAPS), da Secretaria Executiva Regional (SER) IV, no município de Fortaleza (CE). As informações foram investigadas no período de outubro de 2015 a fevereiro de 2016, por meio de um questionário estruturado. A análise dos dados foi calculada através do teste Qui-Quadrado e o Teste Exato de Fisher com 95\% para intervalo de confiança e com significância de $p=0,05$. Participaram do estudo 67 profissionais, sendo 49,2\% $(n=33)$ casados, $35,8 \%(n=24)$ possuem a faixa etária de 31 a 40 anos, e 10,4\% (n = 7) estão satisfeitos com a remuneração salarial. Verificou-se que a remuneração e a fragilidade na organização dos processos de trabalho foram as variáveis com maior índice de insatisfação.

PALAVRAS-CHAVE: Estratégia Saúde da Família; Satisfação no Emprego; Sistema Único de Saúde; Sistemas de Saúde.

\section{SATISFACTION OF PROFESSIONALS INVOLVED IN FAMILY HEALTH STRATEGY IN FORTALEZA, BRAZIL}

ABSTRACT: Factors that interfere in the satisfaction or non-satisfaction of professional involved in Family Health Strategy are investigated. Transversal and quantitative research was undertaken in twelve First Care Units of the Regional Executive Secretary IV in Fortaleza, Brazil. Data were collected between October 2015 and February 2016 through a structured questionnaire. Data analysis was done by the Chi-square test and Fischer's Exact test at 95\% confidence rate and significance at $\mathrm{p}=0.05$. Sixty-seven professionals participated, with $49.2 \%(\mathrm{n}=33)$ married, 35.8\% $(\mathrm{n}=24)$ within the 31-40 year bracket and $10.4 \%$ ( $=7$ ) are satisfied with their pay packet. Wage and weakness in the organization of the labor process were the variables with the highest non-satisfaction index.

KEY WORDS: Family health Strategy; Satisfaction on work; National Health System; Health Systems.

\section{INTRODUÇÃO}

A satisfação ou insatisfação do profissional nos serviços impac- tará, por muitas vezes, diretamente na assistência direcionada à população ${ }^{1}$. A Estratégia Saúde da Família (ESF) é uma proposta do Ministério da Saúde que busca a reorganização da Atenção Primária a Saúde 
(APS) e a reorientação das práticas profissionais, visando o desenvolvimento de ações preventivas, curativas e de reabilitação, conforme descrito nas diretrizes dispostas na Política Nacional de Atenção Básica (PNAB), nº 2.488 de 21 de outubro de $2012^{2}$.

Atendendo às diretrizes dessa estratégia, a equipe é composta, essencialmente, por um profissional médico, de caráter generalista ou especialista em saúde da família, enfermeiro generalista ou especialista em saúde da família, profissionais de nível técnico: auxiliar ou técnico de enfermagem, e Agentes Comunitários de Saúde (ACS) ${ }^{2}$.

Essa estratégia, implantada primeiramente como Programa Saúde da Família (PSF), foi oficializada em Fortaleza (CE), no ano de 1997, através do Decreto $\mathrm{n}^{\circ}$ 10.104/97. Adotou-se como estratégia administrativa a descentralização da gestão municipal por meio da organização de seis Secretarias Executivas Regionais (SER). Propôs-se uma mudança do modelo assistencial, a fim de contribuir com a melhoria da qualidade de vida da população, cooperando para os debates sobre políticas de saúde, principalmente voltadas a uma reorganização do sistema de saúde 3 .

Em 2006, o PSF passou por algumas alterações, fortalecendo a atenção básica, sendo transformado em ESF. Medidas como a priorização das ações de prevenção e promoção da saúde, substituição do modelo hegemônico e biomédico. Dessa forma, o planejamento de estratégias passou a ser realizado de forma interdisciplinar, a abordagem familiar, tornando a ESF uma prioridade para as políticas sanitárias de atendimento à saúde em Fortaleza ${ }^{4,5}$.

Nesse contexto, após a reorganização dos serviços prestados à população, as equipes da ESF confrontaram-se com diversas situações de fragilidades. Dessas, podemos citar as falhas no processo de trabalho da equipe, as dificuldades em compreender as solicitações feitas pelos usuários, os conflitos que podem ocorrer entre profissionais e a gestã $0^{6,7}$.

Por isso, quando se analisa o processo de trabalho, as equipes da ESF se deparam com o confronto entre a representação idealizada da estratégia, as suas diligências pessoais e as necessidades da comunidade que demandam ações e serviços.

Dessa forma, o modelo de reorientação da aten- ção básica, a ESF, tem vários desafios a serem enfrentados, tais como: a precarização das relações de trabalho associada a fatores como instabilidade trabalhista; complexidade em trabalhar em equipe; excesso de trabalho e salários incompatíveis com o desejo dos profissionais ${ }^{89}$.

Compreende-se que o trabalho em saúde envolve o cuidado humano individual e majoritariamente o coletivo, o que implicam relações entre profissionais e a comunidade. Trabalhar envolve o indivíduo e toda sua subjetividade, podendo resultar em prazer ou em sofrimento ${ }^{6}$.

Visto que esses profissionais do Sistema Único de Saúde (SUS) atuam diretamente com os usuários, e tal função não demanda apenas conhecimentos e habilidades $^{10}$, buscou-se investigar a presença de satisfação nos trabalhadores, já que esta é de fundamental importância para as condições de trabalho dos profissionais de saúde.

Embora o quantitativo de produções acadêmicas ligadas à ESF, observa-se que discussões que envolvem a satisfação dos profissionais de saúde devem ser potencializadas, o que evidencia a importância do presente artigo, objetivando investigar os fatores que interferem na satisfação e na insatisfação dos profissionais da ESF, mais precisamente, na SER IV, do município de Fortaleza (CE).

\section{METODOLOGIA}

O estudo foi aprovado pelo Comitê de Ética e Pesquisa do Hospital Geral de Fortaleza, com o número de registro 1.149.98.2.

Caracteriza-se como uma pesquisa avaliativa, do tipo transversal com abordagem quantitativa, realizada nas 12 Unidades de Atenção Primária a Saúde (UAPS), da SER IV, no município de Fortaleza, no Estado do Ceará. A extensão territorial compreende $34.272 \mathrm{~km}^{2}$, abrange 19 bairros, tem densidade demográfica de 305 mil habitantes, a SER IV foi escolhida por ser a de maior extensão territorial ${ }^{11}$.

As informações foram investigadas nas UAPS, entre os meses de outubro de 2015 a fevereiro de 2016. Para a coleta de dados foi utilizado um questionário estruturado pelos autores, com base em pesquisas ${ }^{1,6,7,10}$ que abordam a temática. O questionário abordou doze questões 
sobre o perfil socioeconômico, satisfação profissional, (Conclusão) quanto à divulgação dos indicadores e resultados alcançados pela equipe, sendo eles: a remuneração, educação permanente, equipamentos, estrutura e manutenção da unidade.

Quanto aos critérios de elegibilidade adotou-se para aqueles que se encontravam no exercício de suas atividades no período da coleta e que assinaram o Termo de Consentimento Livre e Esclarecido (TCLE). A abordagem aos sujeitos ocorreu de forma direta, com apresentação do gestor da UAP inicialmente e em seguida o pesquisador daquela Unidade se apresentava, explicava os objetivos e em seguida o Profissional aceitando a coleta de dados ocorria.

Já para os critérios de exclusão, se deu por aqueles que desistissem da pesquisa, com menos de um ano de exercício profissional durante a coleta de dados.

Participaram do estudo 67 profissionais. Para análise estatística dos achados foram calculadas frequências absolutas e relativas para todas as variáveis categóricas. Foi utilizado o teste Qui-Quadrado e o Teste Exato de Fisher com $95 \%$ para intervalo de confiança e com significância de $\mathrm{p}=0,05$.

\section{RESULTADOS}

CARACTERÍSTICAS SOCIOECONÔMICAS DOS PROFISSIONAIS

Tabela 1. Descrição dos achados quanto ao perfil socioeconômico dos profissionais da UAPS. Fortaleza (CE), 2018

(Continua)

\begin{tabular}{|llcc|}
\hline Variáveis & Categorias & $\begin{array}{c}\text { Frequência } \\
(\mathbf{n}=\mathbf{6 7})\end{array}$ & \begin{tabular}{c} 
(\%) \\
\hline Faixa etária
\end{tabular} \\
& 18 a 30 anos & 12 & 17,9 \\
& 31 a 40 & 24 & 35,8 \\
& 41 a 50 & 22 & 32,8 \\
\hline & 61 anos ou mais. & 1 & 1,5 \\
\hline Estado civil & & & \\
& Casados & 33 & 49,2 \\
& Solteiros & 27 & 40,3 \\
\hline
\end{tabular}

\begin{tabular}{|c|c|c|c|}
\hline Variáveis & Categorias & $\begin{array}{c}\text { Frequência } \\
(\mathrm{n}=67)\end{array}$ & (\%) \\
\hline \multicolumn{4}{|c|}{ Estado civil } \\
\hline & $\begin{array}{l}\text { Separado ou divor- } \\
\text { ciado }\end{array}$ & 5 & 7,5 \\
\hline & Não responderam & 2 & 3,0 \\
\hline \multicolumn{4}{|c|}{ Escolaridade } \\
\hline & Nível médio & 35 & 52,2 \\
\hline & Graduação & 11 & 16,4 \\
\hline & Especialização & 17 & 25,4 \\
\hline & Mestrado & 2 & 3,0 \\
\hline & Não responderam & 2 & 3,0 \\
\hline \multicolumn{4}{|c|}{ Renda financeira } \\
\hline & 1-3 salários mínimos & 32 & 47,8 \\
\hline & 4-10 salários mínimos & 16 & 23,9 \\
\hline & Mais de 10 salários & 18 & 26,8 \\
\hline & Não responderam & 1 & 1,5 \\
\hline \multicolumn{4}{|c|}{ Composição Familiar } \\
\hline & 1 filho & 20 & 29,8 \\
\hline & 2 filhos & 18 & 26,9 \\
\hline & 3 filhos & 5 & 7,5 \\
\hline & $4 \mathrm{ou}+$ & 3 & 4,5 \\
\hline
\end{tabular}

Fonte: Dados da própria pesquisa.

Em números, quantitativos e proporcionais, os médicos correspondem a 7,5\% $(n=5)$ do total da amostra, dos quais $20 \%(n=1)$ eram homens; enfermeiras $20,9 \%(n=14)$, todas do sexo feminino; técnicos de enfermagem somam 17,9\% ( $\mathrm{n}=12)$, sendo $83,33 \%$ ( $=10$ ) do sexo feminino; os auxiliares de enfermagem representaram $6,0 \%(n=4)$, as mulheres correspondem a $75 \%(n=3)$; os agentes comunitários 44,8\% ( $n=30)$, sendo $73,33 \%(n=22)$ equivalente às mulheres; e dois profissionais de saúde, correspondente a 3\% da amostra, não puderam ser identificados, pois não marcaram a opção nesse item do questionário. Os profissionais do sexo feminino são maioria, sendo 80,6\% $(n=54)$.

Referente à naturalidade dos profissionais, a maioria, 89,5\% ( $\mathrm{n}=60)$ são do Estado do Ceará. 
NÍVEL DE ESCOLARIDADE E REMUNERAÇÃO SALARIAL

Sobre o nível de escolaridade dos profissionais da ESF, representando com $52,2 \%(n=35)$ das pessoas com o nível médio.

Para a variável da renda financeira, 47,8\% ( $\mathrm{n}=$ 32) recebem de 1-3 salários mínimos e 96,9\% ( $\mathrm{n}=31)$ estão insatisfeitos referentes ao valor que recebem; $23,9 \%$ $(\mathrm{n}=16)$ de $4-10$ salários mínimos e $100 \%(\mathrm{n}=67)$ deles estão descontentes no que diz respeito ao honorário; $26,8 \%(n=18)$ obtêm mais de 10 salários, destes $75 \%$ $(\mathrm{n}=5)$ marcaram a opção de inquietação quanto aos vencimentos. $1,5 \%$ dos profissionais que participaram da pesquisa não responderam o item referente ao salário vigente, $p=0,001$.

Desses profissionais somente $10,4 \%(n=7)$ estão satisfeitos com a remuneração; $88,1 \%(\mathrm{n}=59)$ estão insatisfeitos; e $1,5 \%(n=1)$ não responderam a essa pergunta; $(\mathrm{n}=13)$ dos homens mostraram-se insatisfeitos com a remuneração, enquanto $86,8 \%(\mathrm{n}=46)$ das mulheres informaram estar insatisfeitas, $p=0,329$.

Os médicos se mostraram $40 \%(n=2)$ insatisfeitos quanto à remuneração; as enfermeiras 78,6\% ( $\mathrm{n}=$ 11); $91,7 \%(\mathrm{n}=11)$ dos técnicos; $100 \%(\mathrm{n}=4)$ dos auxiliares de enfermagem; e 100\% $(n=30)$ dos agentes comunitários também, $p=0,002$.

\section{EDUCAÇÃO PERMANENTE EM SAÚDE}

Quanto aos cursos de capacitação e treinamentos que são ofertados aos profissionais do serviço, $47,8 \%$ $(\mathrm{n}=32)$ mencionaram estar satisfeitos; $50,7 \%(\mathrm{n}=34)$ insatisfeitos; e 1,5\% $(n=1)$ não responderam à pergunta relacionada à temática.

Categorizando por sexo, 46,2\% ( $n=6)$ dos homens informaram estar insatisfeitos quanto à qualificação ofertada, enquanto $52,8 \%$ das mulheres manifestaram insatisfação, $p=0,666$.

Referente à categoria profissional, $20 \%(\mathrm{n}=1)$ dos médicos, $71,4 \%(n=10)$ das enfermeiras, $50 \%(n$ $=6)$ dos técnicos de enfermagem, $50 \%(n=2)$ dos auxiliares de enfermagem e $46,7 \%(n=14)$ dos agentes comunitários disseram estar insatisfeitos, $p=0,355$.

DIVULGAÇÃO DE INDICADORES E RESULTADOS ALCANÇADOS PELA EQUIPE NA UNIDADE DE SAÚDE

Considerando a divulgação dos resultados e informações das ações realizadas nas Unidades de Atenção Primária à Saúde (UAPS), apenas 43,3\% (n= 29) dos profissionais informaram satisfação.

\section{EQUIPAMENTOS, ESTRUTURA E MANUTENÇÃO DA UNIDADE DE SAÚDE}

Na Tabela 2 se apresentam os números relativos e absolutos referentes aos profissionais que se mostraram satisfeitos e insatisfeitos nos quesitos: equipamentos, estrutura e manutenção da unidade de saúde.

Tabela 2. Distribuição dos fatores quanto aos equipamentos, estrutura e manutenção da unidade de saúde - SMS, Fortaleza (CE), 2015

\begin{tabular}{|c|c|c|c|c|c|c|}
\hline Variáveis & Médicos N (\%) & Enfermeiras N (\%) & $\begin{array}{c}\text { Técnicos de Enfermagem } \\
\text { N (\%) }\end{array}$ & $\begin{array}{c}\text { Auxiliares de Enfer- } \\
\text { magem N (\%) }\end{array}$ & $\begin{array}{c}\text { Agente Comunitário } \\
\text { de Saúde N (\%) }\end{array}$ & $p$ valor \\
\hline \multicolumn{7}{|c|}{ Equipamentos } \\
\hline Satisfeitos & $4(80)$ & $12(85,7)$ & $10(83,3)$ & $4(100)$ & $22(73,3)$ & \multirow{2}{*}{0,840} \\
\hline Insatisfeitos & $1(20)$ & $2(14,3)$ & $2(16,7)$ & $0(0)$ & $8(26,7)$ & \\
\hline \multicolumn{7}{|l|}{ Estrutura } \\
\hline Satisfeitos & $4(80)$ & $10(71,4)$ & $6(50)$ & $1(25)$ & $13(44,8)$ & 0,280 \\
\hline Insatisfeitos & $1(20)$ & $4(28,6)$ & $6(50)$ & $3(75)$ & $17(55,2)$ & \\
\hline \multicolumn{7}{|l|}{ Manutenção } \\
\hline Satisfeitos & $1(20)$ & $6(46,2)$ & $3(25)$ & $0(0)$ & $6(21,4)$ & 0,398 \\
\hline Insatisfeitos & $4(80)$ & $8(53,8)$ & $9(75)$ & $5(100)$ & $24(78,6)$ & \\
\hline
\end{tabular}

Fonte: Dados da própria pesquisa. 


\section{DISCUSSÃO}

Pesquisas que delimitam as diferentes percepções que englobam a satisfação dos profissionais da saúde, principalmente dos serviços públicos, permitem uma investigação avaliativa completa e fidedigna quanto ao modelo de gestão nos serviços de saúde, pois através dos níveis de satisfação dos profissionais é possível medir a qualidade do atendimento, já que são fatores associados aos serviços prestados à comunidade ${ }^{12}$.

Entende-se, ainda, satisfação como um estado emocional prazeroso que resulta de múltiplos aspectos do trabalho e que pode ser influenciado pela subjetividade de cada indivíduo, vivências e características singulares de cada trabalhador, implicando formas diferenciadas de enfrentamento dos problemas e a tomada cotidiana de decisões ${ }^{13}$.

\section{CARACTERÍSTICAS SOCIODEMOGRÁFICAS DOS PRO- FISSIONAIS}

Em um estudo realizado em Portugal, com médicos e enfermeiros em 2009, verificou-se que as mulheres estão mais propensas a situações de estresse, pois elas sentem-se desvalorizadas, experimentam excesso de trabalho, instabilidade profissional, falta de reconhecimento, problemas familiares e baixa remuneração, influenciando, assim, o estado de insatisfação no ambiente laboral ${ }^{14}$.

Corroborando o estudo supracitado, a presente análise identifica maiores porcentagens de insatisfação no trabalho entre o sexo feminino. Com isso, é possível presumir que a insatisfação das mulheres está relacionada a fatores que configuram a sua vida pessoal com as atribuições do trabalho, havendo uma maior sobrecarga de atividades quando comparada à dos homens, que socialmente desempenham atribuições menores nas atividades domésticas e familiares ${ }^{15}$. Mesmo que as mulheres exerçam um serviço formal, ou seja, remunerado, elas também fazem a maior parte das funções domésticas, tornando a rotina bem mais intensa, cooperando, dessa forma, para o estado de insatisfaçãa ${ }^{16}$.

\section{NÍVEL DE ESCOLARIDADE E REMUNERAÇÃO}

Acredita-se que a formação acadêmica e a titulação profissional são fatores que podem condicionar a insatisfação profissional quando o indivíduo não exerce as suas funções diárias com os seus conhecimentos e habilidades prévias. Uma vez que a remuneração na ESF varia mediante o município e, com isso, alguns valores podem ficar abaixo do esperado, então muitos dos funcionários percebem essa condição como desvalorização e atribuem ao seu cotidiano insatisfação.

Visto no estudo de Ronzani e Silva ${ }^{17}$, a possibilidade de talvez ser os motivos que muitos profissionais atuantes na UAPS e que apresentaram a formação acadêmica voltada para área não a fim, fazendo com que o conhecimento na atenção básica seja resultado da vivência e da aproximação dos profissionais com o grupo em foco ${ }^{17}$. Essa é uma das debilidades do serviço.

Sabendo que o bem-estar relaciona aos aspectos biopsicossociais que interferem o modo como o profissional interage com outras pessoas e em seu meio de trabalho, para que, de fato, os profissionais de saúde consigam atender às necessidades dos usuários, é necessário que tenham condições de trabalho adequadas e uma cultura organizacional livre de retaliações ${ }^{18}$.

Um estudo ${ }^{19}$ desenvolvido com ACS sobre a satisfação e engajamento no trabalho mostrou que o grau de importância da remuneração para a satisfação no trabalho é de $61 \%$, e citou que $24 \%$ dos participantes estão satisfeitos. Diferentemente do nosso estudo, que mostra que $96,9 \%$ estão insatisfeitos com a remuneração.

A partir deste estudo, observou-se que a maioria dos profissionais, independente da escolaridade e categoria profissional, que atuam na atenção básica, está insatisfeita com a remuneração. Visto o elevado nível de insatisfação dos profissionais pesquisados, expõe-se a necessidade de políticas que visem melhor remuneração destes, haja vista que eles lidam diretamente com pessoas em situação de adoecimento as quais necessitam de atenção e cuidado e almejam ter um atendimento de excelência.

Nesta pesquisa detectou-se que dentre os pesquisados destacavam-se, em número, os profissionais que possuem ensino médio, que são em sua maioria Agentes Comunitários de Saúde (ACS's). Corroborando com o es- 
tudo $^{19}$ viu-se que é possível vislumbrar as estratégias de fortalecimento e fragilidades dos agentes para o melhor aproveitamento de seu potencial e melhorias no processo de trabalho por meio da educação permanente.

\section{EDUCAÇÃO PERMANENTE EM SAÚDE}

No estudo realizado em $2006^{20}$, pode-se identificar que profissionais motivados e qualificados são fundamentais para o aumento da produtividade e da qualidade dos serviços prestados à comunidade, a fim de contribuir para a execução das diretrizes propostas pelo SUS.

Nesta pesquisa, observou-se que há satisfação entre os médicos no que diz respeito à capacitação e ao treinamento para o trabalho na ESF. Entretanto, um grande número de enfermeiras informou estar insatisfeito nesse processo. Já os ACS's, o grupo de profissionais com maior número entre os pesquisados, apresenta certo equilíbrio nos níveis de satisfação quanto ao treinamento e cursos de capacitação.

Conforme se pode perceber, existe um índice de insatisfação importante na pesquisa realizada na SER IV, por isso, faz-se necessário que seja elevada a oferta de cursos que possam qualificar os profissionais de saúde que atendem à comunidade.

A capacitação, os processos de educação permanente em saúde e o preparo profissional, manifestam-se como oportunidade para a melhoria salarial e valorização do trabalhador e, por conseguinte, a melhoria do atendimento à comunidade. Com isso, a ESF ajuda na ampliação do mercado de trabalho, dos campos de atuação e ainda possibilita um espaço de prática para aquisição de experiência com o foco para as ações de promoção, prevenção e reabilitação à saúde ${ }^{21}$.

\section{DIVULGAÇÃO DE INDICADORES E RESULTADOS ALCAN- ÇADOS PELA EQUIPE NA UNIDADE DE SAÚDE}

A administração da equipe da ESF possui relativa importância, pois pode afetar diretamente a satisfação dos profissionais e a qualidade dos serviços prestados ${ }^{22}$. Logo, é preciso identificar os pontos positivos e negativos da estratégia para gerar informações necessárias que possam garantir melhorias e auxiliar no avanço da atenção básica no Brasil ${ }^{23}$.

Com isso, observa-se a importância da melhoria por parte das gestões das Unidades de Atenção Primária à Saúde, pois, no estudo realizado, viu-se que 57,7\% dos profissionais estão insatisfeitos com a divulgação dos indicadores e resultados alcançados pela equipe.

Corroborando os dados da pesquisa realizada em Fortaleza, em estudo realizado na África Ocidental em 2006, os profissionais da saúde mostraram-se insatisfeitos com a divulgação dos resultados por parte dos gestores, além de sentirem-se desmotivados com a falta de material. A pesquisa mostrou que, apesar da remuneração ser um fator importante e que não deve ser negligenciado, a divulgação dos resultados da equipe e o reconhecimento pelo trabalho são fatores determinantes para a satisfação desses profissionais ${ }^{20}$.

Portanto, para aumentar a motivação e satisfação dos profissionais, deve-se ter um ambiente que permita a comunicação entre a equipe com prioridade nas soluções dos problemas e divulgação dos resultados alcançados ${ }^{24}$. Além disso, é relevante essa comunicação entre os membros da ESF, para que haja troca de informações sobre os pacientes, o que permite uma tomada de decisões adequadas às necessidades identificadas pela equipe ${ }^{25,5}$.

EQUIPAMENTOS, ESTRUTURA E MANUTENÇÃO DA UNIDADE DE SAÚDE

Para os profissionais, a estrutura física também influencia na qualidade do serviço e, consequentemente, na motivação no trabalho, pois possibilita o desempenho de suas funções de maneira eficiente e atende, assim, às necessidades dos trabalhadores e usuários, apesar de gestores não priorizarem esse item ${ }^{26}$.

Nos locais da realização da pesquisa, a avaliação do Programa de Melhoria ao Acesso e Qualidade da Atenção Básica (PMAQ) apresentou desempenho acima da média quanto à estrutura física e equipamentos da mesma, confirmando, assim, os resultados de que a maioria dos profissionais sente-se satisfeita nesse quesito ${ }^{27}$.

Assim, verificam-se diferentes dimensões do processo de satisfação no trabalho, fortalecendo para a solidificação dos indicadores da gestão de recursos humanos partindo dos fatores promotores da satisfação, identificando esse nível que manifesta pelos grupos de profissionais gerentes e assistenciais que são atuantes nos serviços ${ }^{28}$. 


\section{CONCLUSÃO}

Após a análise dos dados obtidos no presente estudo, detectou-se que vários fatores interferem na satisfação e na motivação no trabalho, sendo a remuneração a variável com maior índice de insatisfação. Além disso, observa-se que quanto menor o nível de escolaridade, mais elevado o nível de insatisfação com o salário.

Conclui-se que para melhorar a satisfação dos profissionais da ESF faz-se necessária mais qualificação e investimento na organização dos processos de trabalho, o que consequentemente acarretará aumento salarial, além de infraestruturas adequadas, capacitação para aprimorar $\mathrm{o}$ atendimento à comunidade e a divulgação dos indicadores de saúde e resultados alcançados pela equipe.

Por fim, cita-se que são necessárias outras pesquisas para aprofundar o conhecimento sobre os fatores de satisfação e insatisfação dos profissionais que atuam na UAPS, visto que estes lidam diariamente com os usuários e devem estar satisfeitos para prestar atendimentos de qualidade à população.

\section{REFERÊNCIAS}

1. Sartoreto IS, Kurcgant P. Satisfação e insatisfação no trabalho do enfermeiro. Revista brasileira de ciências da Saúde. 2017; 21 (2): 181-188.

2. Ministério da Saúde. Secretaria de Atenção à Saúde. Departamento de Atenção Básica. Política Nacional de Atenção Básica. Brasília, 2012.

3. Batista FLR, et al. Políticas Públicas de Saúde em Fortaleza: do movimento pela reforma sanitária à reforma administrativa. Revista do PPG em Políticas Públicas da UECE: O público e o privado, n ${ }^{\circ} 6$ - jul./ dez., 2005.

4. Esmeraldo GROV. A Organização da Estratégia Saúde da Família em Fortaleza - CE: Do Processo de Implantação ao Contexto Atual. Dissertação - Centro de Ciências da Saúde, Universidade Estadual do Ceará, 2009.

5. Oliveira EM, Spiri WC. Programa Saúde da Família: a experiência de equipe multiprofissional. Rev Saude Publica. 2006; 40 (4): 727-33.
6. Lima L, Pires DEP, Forte ECN, Medeiros F. Satisfação e insatisfação no trabalho de profissionais de saúde da atenção básica. Escola Anna Nery Revista de Enfermagem, 2014; 18 (1): 17-24.

7. Brand CI, Antunes RM, Fontana, RT. Satisfações e Insatisfações no Trabalho do Agente Comunitário de Saúde. Cogitare Enferm 2010 jan./mar.; 15 (1): 40-7.

8. Guedes SAG, Oliveira CCC, Albuquerque Jr RLC. Análise da Satisfação dos Profissionais de Saúde da Família com as Condições de Trabalho. Cienc Cuid Saude 2013 jan./mar.; 12 (1): 121-130.

9. Marqui ABT, Jahn AC, Resta DG, Colomé ICS, Zanon T. Caracterização das equipes da Saúde da Família e de seu processo de trabalho. Rev Esc Enferm USP 2010; 44 (4): 956-61.

10. Cotta RMM, Schott M, Azeredo CM, Franceschini SDCC, Priore SE, Dias G. Organização do trabalho e perfil dos profissionais do Programa Saúde da Família: um desafio na reestruturação da atenção básica em saúde. Epidemiol e Serviços Saúde. 2006; 15 (3): 7-18.

11. Prefeitura Municipal de Fortaleza. Secretaria Regional IV. Disponível em: < http://www.fortaleza.ce.gov. br/institucional/a-secretaria-318> Acesso em: 29 jan. 2017.

12. Pelisoli C, Fraga BM, Pereira, CO. Percepções de trabalhadores da saúde pública sobre a rede de atendimento. Mental - Barbacena-MG - jan.jun. 2011; 9 (16): 353-374.

13. Martinez MC, Paraguay AIBB. Satisfação e saúde no trabalho - aspectos conceituais e metodológicos. Cadernos de Psicologia Social do Trabalho. 2003; (6): 59-78.

14. Silva MCM, Gomes ARDS. Stress ocupacional em profissionais de saúde: um estudo com médicos e enfermeiros portugueses. Estud Psicol. 2009; 14 (3): 239-48.

15. Pereira RS, Santos DA, Borges W. A Mulher no Mercado de Trabalho. Programa de Pós-Graduação em Políticas Públicas. Universidade Federal do Maranhão. São Luís Maranhão, 2005. 
16. Daniel C. O trabalho e a questão de gênero: a participação de mulheres na dinâmica do trabalho. O Social em Questão - 2011; 14 (25/26): 323-344.

17. Ronzani TM, Silva C de M. O Programa Saúde da Família segundo profissionais de saúde, gestores e usuários. Ciência \& Saude Colet. 2008;13 (1): 23-34.

18. Tambasco LP, Silva HS, Pinheiro KMK, Gutierrez BAO. A satisfação no trabalho da equipe multiprofissional que atua na Atenção Primária à Saúde. Saúde debate. 2017; 41 (spe2): 140-151.

19. Lino MM, Lanzoni GMM, Albuquerque GL, Schveitzer MC. Perfil socioeconômico, demográfico e de trabalho dos agentes comunitários de saúde. Cogitare Enfermagem. 2012; 17 (2): 57-64.

20. Dieleman M, Toonen J, Touré H, Martineau T. The match between motivation and performance management of health sector workers in Mali. Hum Resour Health. 2006; 4 (2): 1-7.

21. Neto FRGX, Costa MCF, Rocha J, Cunha ICKO. Auxiliares e técnicos de enfermagem na saúde da família: Perfil sociodemográfico e necessidade de qualificação. Trab Educ e Saúde. 2008; 6 (1): 1-14.

22. Nogueira RP. Avaliação de tendências e prioridades sobre recursos humanos de saúde/Roberto Nogueira (coord.) - Brasília: Organização Pan-Americana da Saúde, 2002. (Rede Observatório de Recursos Humanos de Saúde).

23. Perez LG, Sheridan JD, Nicholls AY, Mues KE, Saleme OS, Resend CJ, et al. Professional and community satisfaction with the Brazilian Family health strategy. Rev Saúde Pública 2013; 47 (2): 403-413.

24. Bonenberger M, Aikins M, Akweongo P, Wyss K. The effects of health worker motivation and job satisfaction on turnover intention in Ghana: a cross-sectional study. Hum Resour Health [Internet]. 2014; 12 (1): 43.

25. Peduzzi M. Equipe multiprofissional de saúde: conceito e tipologia. Rev Saúde Pública. 2001; 35 (1): 103-9.

26. Serapioni M, Silva MGS. Avaliação da qualidade do Programa Saúde da Família em municípios do Ceará.
Uma abordagem multidimensional. Ciência \& Saúde Coletiva, 2011; 16 (11): 4315-4326.

27. Ministério da Saúde. Avaliação das UBS - PMAQ. Portal da Saúde. Disponível em: Departamento de Atenção Básica. Brasília. 2012.

28. Siqueira VTA, Kurcgant P. Satisfação no trabalho: indicador de qualidade no gerenciamento de recursos humanos em enfermagem. Rev Esc Enferm USP 2012; 46 (1): 151-7.

Recebido em: 29/03/2018

Aceito em: 20/12/2018 